\title{
Chiral Fluctuations in MnSi above the Curie Temperature
}

\author{
B. Roessli, ${ }^{1}$ P. Böni, ${ }^{2}$ W. E. Fischer, ${ }^{1}$ and Y. Endoh ${ }^{3}$ \\ ${ }^{1}$ Laboratory for Neutron Scattering, ETH Zurich \& Paul Scherrer Institute, CH-5232 Villigen PSI \\ ${ }^{2}$ Physik-Department E21, Technische Universität München, D-85747 Garching, Germany \\ ${ }^{3}$ Institute of Materials Research, Tohoku University, Katahira, Aoba-ku, Sendai, 980-8577, Japan
}

(Received 17 January 2002; published 23 May 2002)

\begin{abstract}
Polarized neutrons are used to determine the antisymmetric part of the magnetic susceptibility in noncentrosymmetric MnSi. The paramagnetic fluctuations are found to be incommensurate with the chemical lattice and to have a chiral character. We argue that antisymmetric interactions must be taken into account to properly describe the critical dynamics in $\mathrm{MnSi}$ above $T_{C}$. The possibility of directly measuring the polarization dependent part of the dynamical susceptibility in a large class of compounds by polarized inelastic neutron scattering is outlined as it can yield evidence for antisymmetric interactions such as spin-orbit coupling in metals as well as in insulators.
\end{abstract}

DOI: 10.1103/PhysRevLett.88.237204

PACS numbers: 75.25.+z, 71.20.Lp, 71.70.Ej

Ordered states with helical arrangement of the magnetic moments are described by a chiral order parameter $\vec{C}=\vec{S}_{1} \times \vec{S}_{2}$, which yields the left- or right-handed rotation of neighboring spins along the pitch of the helix. Examples for compounds of that sort are rare-earth metals such as Ho [1]. Spins on a frustrated lattice form another class of systems, where simultaneous ordering of chiral and spin parameters can be found. In this case, the ground state is highly degenerate and it is possible to obtain two equivalent ground states which differ only by the sense of rotation (left or right) of the magnetic moments from sublattice to sublattice, hence yielding an example of chiral degeneracy. As a consequence of the chiral symmetry of the order parameter, a new universality class results that is characterized by novel critical exponents as calculated by Monte Carlo simulations [2] and measured by neutron scattering [3] in the $X Y$-antiferromagnet $\mathrm{CsMnBr}_{3}$. An interesting but still unresolved problem is the characterization of chiral spin fluctuations that have been suggested to play an important role, e.g., in the doped high- $T_{c}$ superconductors [4]. The measurement of chiral fluctuations is, however, a difficult task and can usually be performed only by projecting the magnetic fluctuations on a field-induced magnetization $[5,6]$.

In this Letter, we show that chiral fluctuations can be directly observed in noncentrosymmetric crystals without disturbing the sample by a magnetic field. We present results of polarized inelastic neutron scattering experiments performed in the paramagnetic phase of the itinerant ferromagnet $\mathrm{MnSi}$ that confirm the chiral character of the spin fluctuations due to spin-orbit coupling and discuss the experimental results in the framework of selfconsistent renormalization theory of spin fluctuations in itinerant magnets [7]. Being a prototype of a weak itinerant ferromagnet with unusual magnetic and transport properties [8], the magnetic fluctuations in $\mathrm{MnSi}$ have been the subject of detailed investigations by means of unpolarized and polarized neutron scattering. The results demonstrate the itinerant nature of the spin fluctuations [9-11] as well as the occurrence of spiral correlations [12] and strong longitudinal fluctuations [13,14].

$\mathrm{MnSi}$ has a cubic space group $P 2{ }_{1} 3$ with a lattice constant $a=4.558 \AA$ lacking a center of symmetry which leads to a ferromagnetic spiral along the $\left[\begin{array}{lll}1 & 1 & 1\end{array}\right]$ direction with a propagation vector $\vec{k}_{0}=$ $(2 \pi / a)(0.017,0.017,0.017)$ yielding a period of approximately $180 \AA$ [15]. The Curie temperature is $T_{C}=29.5 \mathrm{~K}$. The spontaneous magnetic moment of Mn $\mu_{s} \simeq 0.4 \mu_{B}$ is strongly reduced from its free ion value $\mu_{f}=2.5 \mu_{B}$. As shown in the inset of Fig. 1 the four $\mathrm{Mn}$ and $\mathrm{Si}$ atoms are placed at the positions $(u, u, u),\left(\frac{1}{2}+u, \frac{1}{2}-u,-u\right),\left(\frac{1}{2}-u,-u, \frac{1}{2}+u\right)$, and $\left(-u, \frac{1}{2}+u, \frac{1}{2}-u\right)$ with $u_{\mathrm{Mn}}=0.138$ and $u_{\mathrm{Si}}=0.845$, respectively.

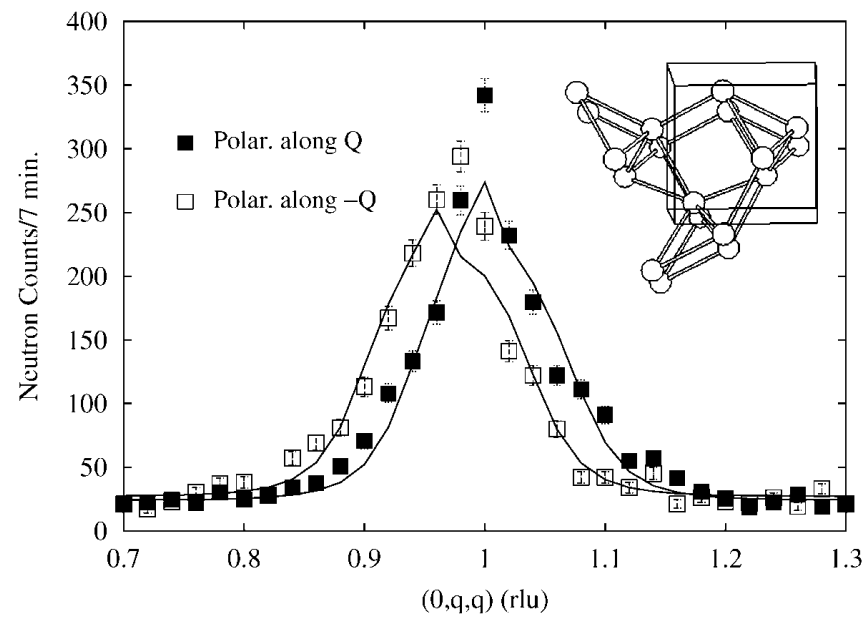

FIG. 1. Inelastic spectra in $\mathrm{MnSi}(\hbar \omega=0.5 \mathrm{meV})$ at $T=$ $31 \mathrm{~K}$ for the neutron polarization parallel and antiparallel to the scattering vector $\vec{Q}$, respectively. The solid lines are fits to the data. The inset shows the crystal structure of MnSi. For means of clarity only the Mn ions are drawn. Note that $\mathrm{MnSi}$ is not centrosymmetric. 
We investigated the paramagnetic fluctuations in a large single domain crystal of $\mathrm{MnSi}$ (mosaic $\eta=1.5^{\circ}$ ) of $10 \mathrm{~cm}^{3}$ on the triple-axis spectrometer TASP at SINQ.

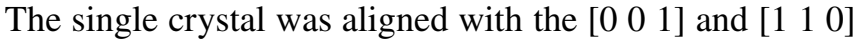
crystallographic directions in the scattering plane. Most constant energy scans were performed in the paramagnetic phase in order to relax the problem of depolarization of the neutron beam in the ordered phase. The spectrometer was operated in the constant final energy mode with a neutron wave vector $\vec{k}_{f}=1.97 \AA^{-1}$ corresponding to an energy resolution at the elastic position of $\sim 0.45 \mathrm{meV}$. In order to suppress contamination by higher order neutrons a pyrolytic graphite filter was installed in the scattered beam. The incident neutrons were polarized by means of a remanent $\mathrm{FeCoV} / \mathrm{TiN}$-type bender that was inserted after the monochromator [16]. The polarization of the neutron beam at the sample position was maintained by a guide field $B_{g}=10 \mathrm{G}$ that defines also the polarization of the neutrons $\vec{P}_{i}$ with respect to the scattering vector $\vec{Q}=$ $\vec{k}_{i}-\vec{k}_{f}$ at the sample position. Such a small field has no influence on magnetic fluctuations as $g \mu_{B} H \ll k_{B} T$.

In contrast to previous experiments, where the polarization $\vec{P}_{f}$ of the scattered neutrons was also measured in order to distinguish between longitudinal and transverse fluctuations [13], we did not analyze $\vec{P}_{f}$, as our goal was to detect a polarization dependent scattering that is proportional to $\sigma_{p} \propto\left(\hat{\vec{Q}} \cdot \vec{P}_{i}\right)$ as discussed below. A typical constant-energy scan with $\hbar \omega=0.5 \mathrm{meV}$ measured in the paramagnetic phase at $T=31 \mathrm{~K}$ is shown in Fig. 1 for the polarization of the incident neutrons $\vec{P}_{i}$ parallel and antiparallel to the scattering vector $\vec{Q}$. It is clearly seen that the peak positions depend on $\vec{P}_{i}$ and appear at the incommensurate positions $\vec{Q}=\vec{\tau} \pm \vec{\delta}$ with respect to the reciprocal lattice vector $\vec{\tau}_{011}$ of the nuclear unit cell. Obviously, this shift of the peaks with respect to $\left(\begin{array}{lll}0 & 1 & 1\end{array}\right)$ would be hardly visible with unpolarized neutrons and could not observed in previous inelastic neutron scattering experiments.

In order to discuss our results, we remind the reader that the scattering function $S(\vec{Q}, \omega)$ is related to the dynamical susceptibility through the fluctuation-dissipation theorem $S(\vec{Q}, \omega)=2 \hbar /[1-\exp (-\hbar \omega / k T)] \operatorname{Im} \chi(\vec{Q}, \omega)$. The general expression for the cross section of magnetic scattering with polarized neutrons reads [17]

$$
\begin{aligned}
S(\vec{Q}, \omega) \sim & \sum_{\alpha, \beta}\left(\delta_{\alpha, \beta}-\hat{Q}_{\alpha} \hat{Q}_{\beta}\right) A^{\alpha \beta}(\vec{Q}, \omega) \\
& +\sum_{\alpha, \beta}\left(\hat{\vec{Q}} \cdot \vec{P}_{i}\right) \sum_{\gamma} \epsilon_{\alpha, \beta, \gamma} \hat{Q}^{\gamma} B^{\alpha \beta}(\vec{Q}, \omega),
\end{aligned}
$$

where $(\vec{Q}, \omega)$ are the momentum and energy transfers from the neutron to the sample, $\hat{\vec{Q}}=\vec{Q} /|\vec{Q}|$, and $\alpha, \beta, \gamma$ indicate Cartesian coordinates. The first term in Eq. (1) is independent of the polarization of the incident neutrons, while the second is polarization dependent through the factor $\left(\hat{\vec{Q}} \cdot \vec{P}_{i}\right) . \quad \vec{P}_{i}$ denotes the direction of the neutron polarization before scattering and its scalar is equal to 1 when the beam is fully polarized. $A^{\alpha \beta}=\frac{1}{2}\left(S^{\alpha \beta}+S^{\beta \alpha}\right)$ and $B^{\alpha \beta}=\frac{1}{2}\left(S^{\alpha \beta}-S^{\beta \alpha}\right)$ are the symmetric and antisymmetric parts of the Fourier transforms of the spin correlation function $\left\langle s_{l}^{\alpha} s_{l^{\prime}}^{\beta}\right\rangle$ with $S^{\alpha \beta}(\vec{Q}, \omega)=\frac{1}{2 \pi N} \int_{-\infty}^{\infty} d t e^{-i \omega t} \sum_{l l^{\prime}} e^{i \vec{Q}\left(\vec{X}_{l}-\vec{X}_{l^{\prime}}\right)}\left\langle s_{l}^{\alpha} s_{l^{\prime}}^{\beta}(t)\right\rangle$. The vectors $\vec{X}_{l}$ designate the positions of the scattering centers in the lattice.

Following Ref. [18] we define now an axial vector $\vec{B}$ by $\sum_{\alpha \beta} \epsilon_{\alpha \beta \gamma} B^{\alpha \beta}=B^{\gamma}(\vec{Q}, \omega)$ that represents the antisymmetric part of the susceptibility. It depends on the neutron polarization as follows:

$$
\left(\hat{\vec{Q}} \cdot \vec{P}_{i}\right)(\hat{\vec{Q}} \cdot \vec{B})
$$

and vanishes for centrosymmetric systems such as $\mathrm{Fe}$ or Ho or when there is no long-range order. In the absence of symmetry breaking fields such as external magnetic fields, uniaxial pressure, etc., similar scans with polarized neutrons would not yield inelastic scattering that depends on the polarization of the neutrons. However, an intrinsic anisotropy of the spin Hamiltonian in a system that lacks lattice inversion symmetry may provide an axial interaction leading to a polarization dependent cross section. The polarization dependent scattering of the present experiments is therefore an indication of fluctuations with chiral signature and points towards the existence of an axial vector $\vec{B}$ that is not necessarily commensurate with the lattice. Hence, according to Eq. (2) the neutron scattering function in $\mathrm{MnSi}$ contains a nonvanishing antisymmetric part.

Because the crystal structure of $\mathrm{MnSi}$ is noncentrosymmetric and the magnetic ground state forms a helix with spins perpendicular to the [ $\left[\begin{array}{lll}1 & 1 & 1\end{array}\right]$ crystallographic direction, it is reasonable to interpret the polarization-dependent transverse part of the dynamical susceptibility in terms of the Dzyaloshinskii-Moriya (DM) interaction [19,20] similarly as it was done in other noncentrosymmetric systems that show incommensurate ordering [21,22].

Usually the DM interaction is written as the cross product of interacting spins $H_{\mathrm{DM}}=\sum_{l, m} \vec{D}_{l, m} \cdot\left(\vec{s}_{l} \times \vec{s}_{m}\right)$, where the direction of the DM vector $\vec{D}$ is determined by bond symmetry and its scalar by the strength of the spin-orbit coupling [20]. Although the DM interaction was originally introduced on microscopic grounds for ionic crystals, it was shown that antisymmetric spin interactions are also present in metals with noncentrosymmetric crystal symmetry [23]. In this case, the free energy contains at term linear in $\vec{k}_{0}$ of the form $D\left[\vec{S}_{1} \times \vec{S}_{2}\right] \cdot \vec{k}_{0}$. Here, the sign of $D$ defines the helicity of the spin fluctuations, i.e., the helicity of the spiral, and the direction of the wave vector of the spiral $\vec{k}_{0}$ is determined by the crystalline anisotropy and the dipolar interactions [24]. Well above $T_{C}$, the anisotropy disappears and the dipolar interactions are so weak that the spin fluctuations become isotropic. 
Hence, in a similar way as for insulators with localized spin densities, the antisymmetric interactions originate from the spin-orbit coupling in the absence of an inversion center and a finite contribution to the antisymmetric part of the wave-vector dependent dynamical susceptibility is obtained.

For the case of a uniform DM interaction with $\vec{D}=$ $D \vec{k}_{0}$, the transverse part of the neutron scattering function $S^{\perp}(\vec{Q}, \omega)$ depends on the polarization of the neutron beam [25] as follows:

$$
\begin{aligned}
S^{\perp}\left(\vec{P}_{i}, \vec{Q}, \omega\right)= & \frac{2 \hbar}{1-\exp \left(-\hbar \omega / k_{B} T\right)} \\
& \times \operatorname{Im}\left\{\left[1+(\hat{\vec{D}} \cdot \hat{\vec{Q}})\left(\hat{\vec{Q}} \cdot \vec{P}_{i}\right)\right] \chi^{\perp}[\vec{q}(-\delta), \omega]\right. \\
& \left.+\left[1-(\hat{\vec{D}} \cdot \hat{\vec{Q}})\left(\hat{\vec{Q}} \cdot \vec{P}_{i}\right)\right] \chi^{\perp}[\vec{q}(\delta), \omega]\right\} .
\end{aligned}
$$

Here, $\vec{q}$ designates the reduced momentum transfer with respect to the nearest magnetic Bragg peak at $\vec{\tau} \pm \vec{\delta}$. Equation (3) shows that the cross section for $\pm \vec{P}_{i} \perp \vec{Q}$ is indeed independent of $\vec{P}_{i}$ as observed in Fig. 2. For parallel alignment of $\hat{\vec{D}}, \hat{\vec{Q}}, \pm \hat{\vec{P}}_{i}$, only fluctuations around the position $(-\delta)$, respectively $(\delta)$ contribute to the scattering. By subtracting the inelastic spectra taken with $\vec{P}_{i}$ parallel and antiparallel to $\vec{Q}$, the polarization dependent part of the cross section can be isolated, as demonstrated in Fig. 3 for two temperatures $T=31$ and $T=40 \mathrm{~K}$.

Close to $T_{C}$, the intensity is rather high and the crossing at $\vec{Q}=\left(\begin{array}{lll}0 & 1 & 1\end{array}\right)$ is sharply peaked. At $40 \mathrm{~K}$ the intensity becomes small and the transition at $\left(\begin{array}{lll}0 & 1 & 1\end{array}\right)$ becomes smooth indicating the decreases of the correlation length with increasing temperature. In order to characterize the $q$ dependence of the chiral correlations in more detail

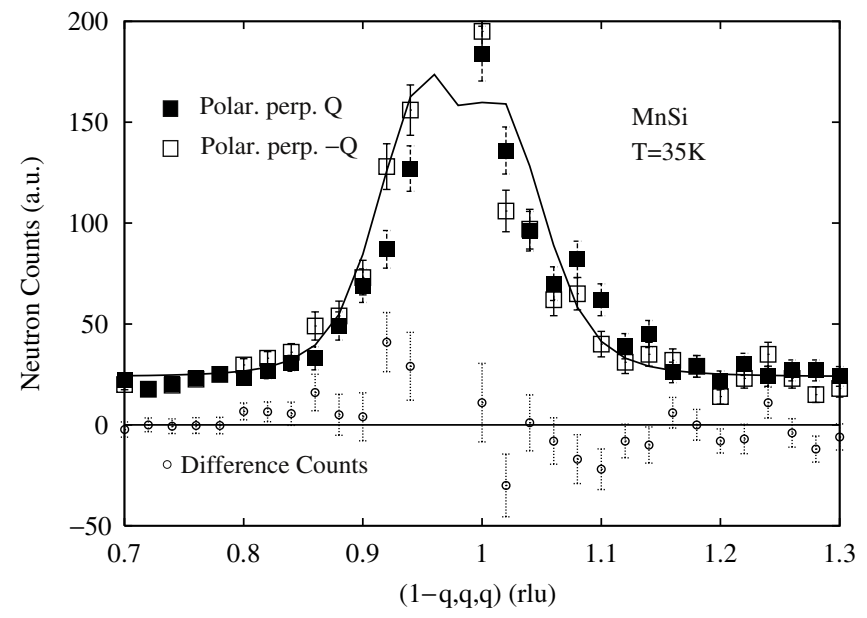

FIG. 2. Neutron spectra in MnSi for an energy transfer $\hbar \omega=$ $0.5 \mathrm{meV}$ as measured at $T=35 \mathrm{~K}$ for $\pm \vec{P}_{i}$ perpendicular to $\vec{Q}$, respectively. The solid line shows a fit to the data and the small symbols represent the difference signal that is independent of $\vec{P}_{i}$. See text for details. we have measured the polarization-dependent part of $S(\vec{Q}, \omega=0.5 \mathrm{meV})$ in the vicinity of the $\left(\begin{array}{lll}0 & 1 & 1\end{array}\right)$ Bragg peak at $T=35 \mathrm{~K}$. The contour plot (Fig. 4) indicates that the DM interaction vector in $\mathrm{MnSi}$ has a component along the [0 $\left.\begin{array}{lll}0 & 1 & 1\end{array}\right]$ crystallographic direction inducing paramagnetic fluctuations that are centered at positions incommensurate with the chemical lattice.

In order to proceed further with the analysis we assume for the transverse susceptibilities in Eq. (3) the expression for itinerant magnets as given by self-consistent renormalization theory (SCR) [7]

$$
\operatorname{Im} \chi^{\perp}[\vec{q}( \pm \delta), \omega]=\chi^{\perp}[\vec{q}( \pm \delta)] \frac{\kappa_{\delta}^{2}}{q^{2}+\kappa_{\delta}^{2}} \frac{\omega \Gamma}{\omega^{2}+\Gamma^{2}},
$$

with $\kappa_{\delta}$ the inverse correlation length. For itinerant ferromagnets the damping of the spin fluctuations is given by $\Gamma_{\vec{q}( \pm \delta)}=u q\left(q^{2}+\kappa_{\delta}^{2}\right)$ with $u=u(\delta)$ reflecting the damping of the spin fluctuations. Experimentally, it has been found from previous inelastic neutron scattering measurements that the damping of the low-energy fluctuations in $\mathrm{MnSi}$ is adequately described using the results of the SCR theory rather than the $q^{z}(z=2.5)$ wave-vector dependence expected for a Heisenberg magnet [10].

The solid lines of Figs. 1-3 show fits of $S(\vec{Q}, \omega)$ to the polarized beam data. It is seen that the form of the dynamical susceptibility for itinerant magnets reproduces the data well if the incommensurability is properly taken into account. Using Eqs. (3) and (4) and taking into account the resolution function of the spectrometer, we extract values $\kappa_{0}=0.12 \AA^{-1}$ and $u=27 \mathrm{meV} \AA^{3}$ that are in reasonable agreement with the analysis given in Ref. [11].

The positions of maximum and minimum intensity of the critical scattering shown in Fig. 4 are located near $\delta \sim$ $(0,0.02,0.02)$ at $T=35 \mathrm{~K}$ and not at the incommensurate

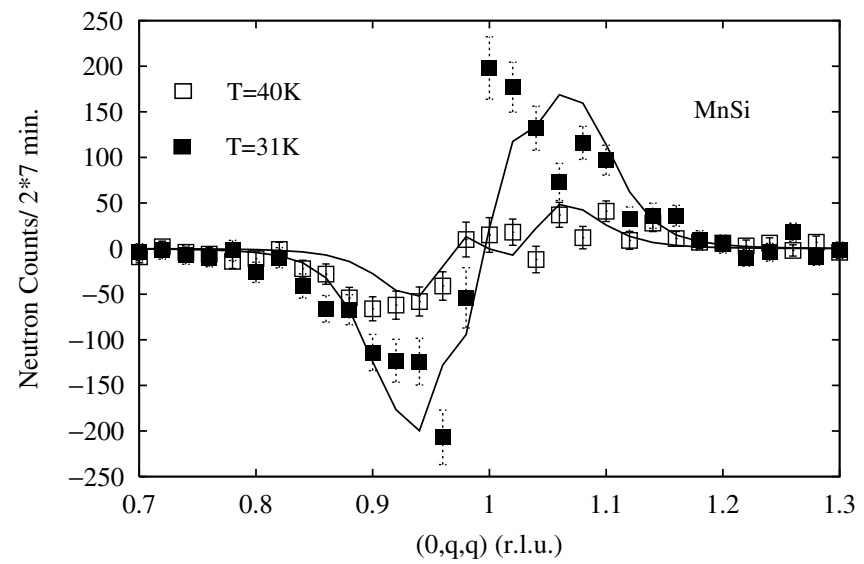

FIG. 3. Difference neutron counts for polarization $\vec{P}_{i}$ of the incident neutron beam parallel and antiparallel to $\vec{Q}$ in $\mathrm{MnSi}$ at $T=31$ and $40 \mathrm{~K}$, respectively. The solid lines are fit to the data using the SRC result for the dynamical susceptibility with the parameters given in the text. 


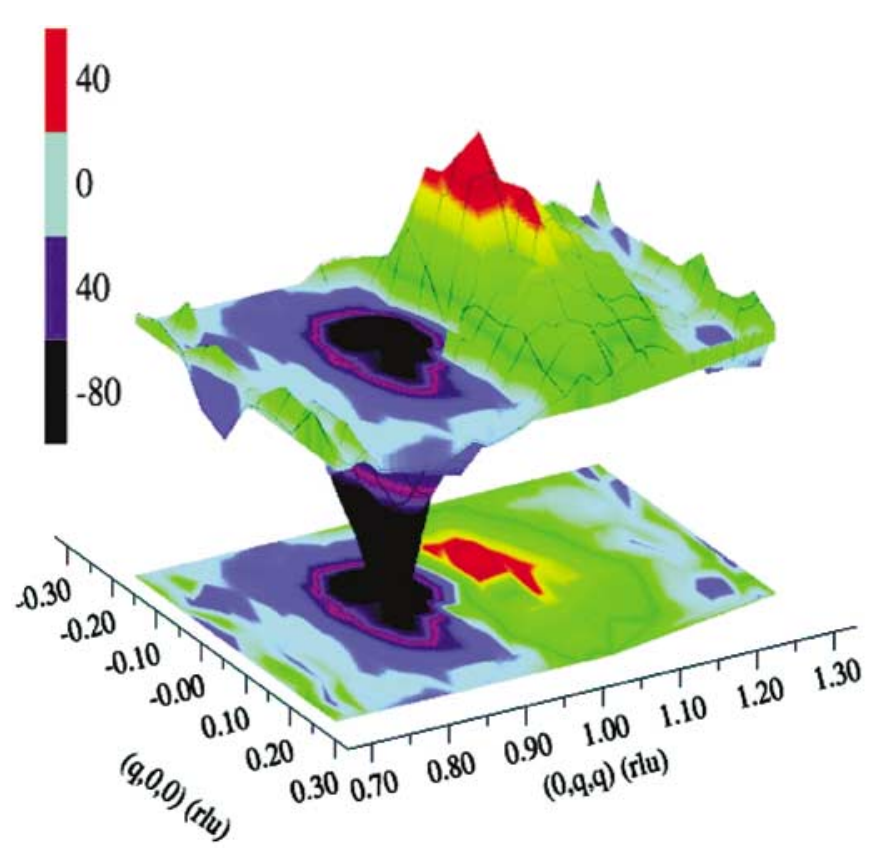

FIG. 4 (color). Contour map of the polarization dependent scattering for an energy transfer $\hbar \omega=0.5 \mathrm{meV}$ as measured near the $\left(\begin{array}{lll}0 & 1 & 1\end{array}\right)$ reciprocal lattice point at $T=35 \mathrm{~K}$.

positions of the magnetic Bragg peaks in the ordered phase. We find that the extrema of $\operatorname{Im} \chi^{\perp}(\vec{q}, \omega)$ lie on a sphere with a radius corresponding approximately to the pitch $\left|\vec{k}_{0}\right|$ of the magnetic spiral in the ordered phase. This result proves that the direction of the propagation vector of the spiral $\vec{k}_{0}$ is determined only by anisotropies that are lost above $T_{C}$ [24].

In addition, the data show that the $T$ dependence of the position of the critical scattering is determined by the increase of $\kappa_{\delta}$ with increasing $T$ according to $\kappa_{\delta}(T)=$ $\kappa_{0}\left(T / T_{C}-1\right)^{0.5}[10]$ that describes our measurements well. We note that the mean-field-like value for $\nu$ is close to the expected exponent $\nu=0.55$ for chiral symmetry [2]. In conclusion, we have shown that chiral fluctuations can be measured by means of polarized inelastic neutron scattering in zero field, when the antisymmetric part of the dynamical susceptibility has a finite value. We have shown that this is the case in metallic MnSi that has a noncentrosymmetric crystal symmetry. Here, the axial interaction leads to a polarization dependent neutron cross section that originates from the DM interaction. Above $T_{C}$, the magnetic anisotropy vanishes and the maximum of magnetic scattering is located on spheres around the nuclear Bragg peaks. Similar investigations of the polarization dependent part of the susceptibility can be performed in a large class of other physical systems. They will yield direct evidence for the presence of antisymmetric interactions in magnetic insulators with DM interactions, high- $T_{c}$ superconductors (e.g., $\mathrm{La}_{2} \mathrm{CuO}_{4}$ [26]), nickelates [27], quasi-onedimensional antiferromagnets [28] or metallic compounds such as FeGe [29].

[1] V. P. Plakhty et al., Phys. Rev. B 64, 100402(R) (2001).

[2] H. Kawamura, Phys. Rev. B 38, 4916 (1988).

[3] T. E. Mason et al., Phys. Rev. B 39, 586 (1989).

[4] P. E. Sulewski et al., Phys. Rev. Lett. 67, 3864 (1991).

[5] S. V. Maleyev, Phys. Rev. Lett. 75, 4682 (1995).

[6] V. P. Plakhty et al., Europhys. Lett. 48, 215 (1999).

[7] T. Moriya, in Spin Fluctuations in Itinerant Electron Magnetism (Springer-Verlag, Berlin, 1985), Vol. 56.

[8] C. Pfleiderer et al., Nature (London) 414, 427 (2001).

[9] Y. Ishikawa et al., Phys. Rev. B 16, 4956 (1977).

[10] Y. Ishikawa et al., Phys. Rev. B 25, 254 (1982).

[11] Y. Ishikawa et al., Phys. Rev. B 31, 5884 (1985).

[12] G. Shirane et al., Phys. Rev. B 28, 6251 (1983).

[13] S. Tixier et al., Physica (Amsterdam) 241B-243B, 613 (1998).

[14] F. Semadeni et al., Physica (Amsterdam) 267B-268B, 248 (1999).

[15] Y. Ishikawa et al., Solid State Commun. 19, 525 (1976).

[16] F. Semadeni et al., Physica (Amsterdam) 297B, 152 (2001).

[17] E.g., Yu A. Izyumov, Sov. Phys. Usp. 27, 845 (1984).

[18] S. W. Lovesey and E. Balcar, Physica (Amsterdam) 267B-268B, 221 (1999), and references therein.

[19] L. Dzyaloshinskii, J. Phys. Chem. Solids 4, 241 (1958).

[20] T. Moriya, Phys. Rev. 120, 91 (1960).

[21] A. Zheludev et al., Phys. Rev. Lett. 78, 4857 (1997).

[22] B. Roessli et al., Phys. Rev. Lett. 86, 1885 (2001).

[23] M. Kataoka et al., J. Phys. Soc. Jpn. 53, 3624 (1984).

[24] M. Kataoka and O. Nakanishi, J. Phys. Soc. Jpn. 50, 3888 (1981).

[25] D. N. Aristov and S. V. Maleyev, Phys. Rev. B 62, R751 (2000).

[26] J. Berger and A. Aharony, Phys. Rev. B 46, 6477 (1992).

[27] W. Koshibae, Y. Ohta, and S. Maekawa, Phys. Rev. B 50, 3767 (1994).

[28] I. Tsukada et al., Phys. Rev. Lett. 87, 127203 (2001).

[29] B. Lebech, J. Bernhard, and T. Freltoft, J. Phys. Condens. Matter 1, 6105 (1989). 